\title{
Effect of MeJA and Light on the Accumulation of Betulin and Oleanolic Acid in the Saplings of White Birch (Betula platyphylla Suk.)
}

\author{
Jing Yin ${ }^{1 *}$, Hongsi Ma ${ }^{1}$, Yuan Gong ${ }^{1}$, Jialei Xiao ${ }^{2}$, Lichao Jiang ${ }^{1}$, Yaguang Zhan ${ }^{1 *}$, Chunxiao Li ${ }^{1}$, \\ Chunlin Ren ${ }^{1}$, Yuanbiao Yang ${ }^{3}$
}

${ }^{1}$ College of Life Science, Northeast Forestry University, Harbin, China; ${ }^{2}$ Heilongjiang Academy of Agricultural Sciences, Harbin, China; ${ }^{3}$ Maoershan Experimental Forestry Center, Northeast Forestry University, Harbin, China.

Email: *yinjing20135@163.com, *yaguangzhan@126.com

Received July $29^{\text {th }}, 2013$; revised August $3^{\text {rd }}, 2013$; accepted September $17^{\text {th }}, 2013$

Copyright (C) 2013 Jing Yin et al. This is an open access article distributed under the Creative Commons Attribution License, which permits unrestricted use, distribution, and reproduction in any medium, provided the original work is properly cited.

\begin{abstract}
In this study, we investigated the effect of different types of light and MeJA treatment on the accumulation of betulin and oleanolic acid in various organs of white birch. Our results showed that betulin and oleanolic were accumulated mainly in the stalk skin. The content of both substances in the stalk skin was significantly affected by seasons with a peak accumulation in August. The content of oleanolic and betulin was significantly decreased in the stem skin treated with 4 types of light (red, yellow, blue and green) compared with the plant with normal illumination. In contrast, oleanolic acid in leaves was increased by 13.28 folds when the white birch was treated with green light. Betulin was increased by 1.959 folds in leaves of white birch treated with blue light. The highest content of betulin and oleanolic acid in various organs of birch with appropriate shading treatment (light transmittance: 50\%) was increased by $45.09 \%$ and $30.50 \%$, respectively, in comparison with those with non-shading treatment. Content of oleanolic acid and betulin can be significantly improved in various parts of birch after treatment with different concentration of MeJA. The study lays the foundation to metabolic regulation of oleanolic acid and betulin in birch.
\end{abstract}

Keywords: Birch (Betula platyphylla Suk.); Betulin; Oleanolic Acid; Light; MeJA

\section{Introduction}

Betulin and oleanolic, widely distributed in the plant kingdom, are pentacyclic triterpenes compounds, belonging to lupane and oleanane type, respectively. The richest biosource of betulin is the outer bark of birch (Betula platyphylla suk.). In addition, the content of betulin in birch is affected by the age, variety, growing season and environment of the plant. Thus, the content of betulin ranges from $10 \%$ to $40 \%$ of the total mass of birch. Betulin exhibits a broad range of biological and medicinal properties, for example, anticancer [1,2], antitumoral [3,4], anti-inflammatory [5], anti-HIV[6-8], antimalarial, hepatoprotective, antiplasmodial, lipid-lowering, choleretic and anti-feedant activities [7,9]. Oleanolic acid possesses many similar important biological activities, for example, anti-inflammatory [10], anti-hyperlipidemic

${ }^{*}$ Corresponding authors.
[11-12], antiulcer [13], antioxidant activity [14] antitumor [15] and hepatoprotective activities. Oleanolic acid can also decrease the blood lipids and improve the immunity. Because oleanolic acid and betulin are natural products of plants, they can selectively inhibit tumor cell growth with low toxicity on normal cells. Therefore, these substances can be developed into novel drugs with broad clinical applications. Betulin is currently undergoing clinical trials at the National Cancer Institute (NCI).

Synthesis of secondary metabolites is regulated by various biotic and abiotic factors. Light plays an important role in the accumulation of plant secondary metabolites. Light is an important factor affecting growth, organogenesis and the formation of plant products including both primary and secondary metabolites. The stimulatory effect of light on the formation of compounds, including terpenoid, flavonoid and anthocyanins has been shown in plants [16-18]. In addition to the earlier men- 
tioned effects of light on the production of plant secondary metabolites, light is also involved in the regulation of secondary product secretion [19]. In addition, studies have proved that endogenous elicitors methyl jasmonate (MeJA) can not only induce plant stress resistance, anti-worms and microbial infection and plant defense system [20,21], but also promote the synthesis of secondary metabolites. MeJA is considered as a very effective elicitor in promoting the induction of key enzymes involved in the synthesis of paclitaxel, camptothecin, isoflavones and licorice saponins [22-25]. MeJA can also induce the production of many monoterpene and terpenoid synthetic resin of the spruce, pine and coconut to resist pathogen infection and grave-sweepers [26].

Previous studies on triterpenes focused mainly on the synthesis, extraction and pharmacological analysis of betulin $[27,28]$. Few studies investigated the accumulation and regulation of the triterpenes in the saplings of white birch during the growing seasons. In this study, we used 1-year-old white birch to study the effect of light and MeJA treatment on the accumulation of oleanolic acid and betulin. We expected to reveal the synthesis and regulation mechanism of betulin and oleanolic acid in white birch.

\section{Results and Discussion}

\subsection{The Effect of Different Types of Light on the Triterpene Accumulation in White Birch}

Four types of light did not facilitate the accumulation of oleanolic acid in stem skin. Oleanolic acid was not de- tected in the stem bark of birch treated with red light. In contrast, red light significantly increased the oleanolic acid (up to $2.626 \mathrm{mg} \cdot \mathrm{g}^{-1}$ ) accumulation in root bark by $49.12 \%$ compared with the control plants (Figure 1(a)). The other 3 types of light did not promote oleanolic acid accumulation in the root bark. All the 4 kinds of light promoted the accumulation of oleanolic acid in leaves. The level of oleanolic acid in leaves of green lighttreated plants was $3.184 \mathrm{mg} \cdot \mathrm{g}^{-1}$ (DW), which was 13.28 times of the control plants. After treatment with green light, the total content of oleanolic acid in leaves, root bark and stem skin of white birch saplings was significantly increased. In contrast, other three kinds of light treatment significantly reduced the total oleanolic acid content in the stem skin. Oleanolic acid content was the minimum in various parts of birch treated with blue light (Figure 1(a)). Betulin was can be detected in leaves and root bark of birch saplings, but the level was significantly lower than that in the skin stem. None of the 4 lights can increase the betulin accumulation in skin stem and root bark of birch saplings. Green light slightly decreased the synthesis of betulin in leaves, while the red, yellow and blue light can increase the content of betulin in the leaves of birch. The content of betulin in the leaves of blue light-treated birch was 1.959 times of the control plants (Figure 1(b)).

Many medicinal plants contain a variety of triterpenoid distributed in different tissues. For example, studies showed that more than 60 types of triterpenoids were contained in licorice and licorice saponins that are mainly present in the roots and xylem. Betulinic acid was

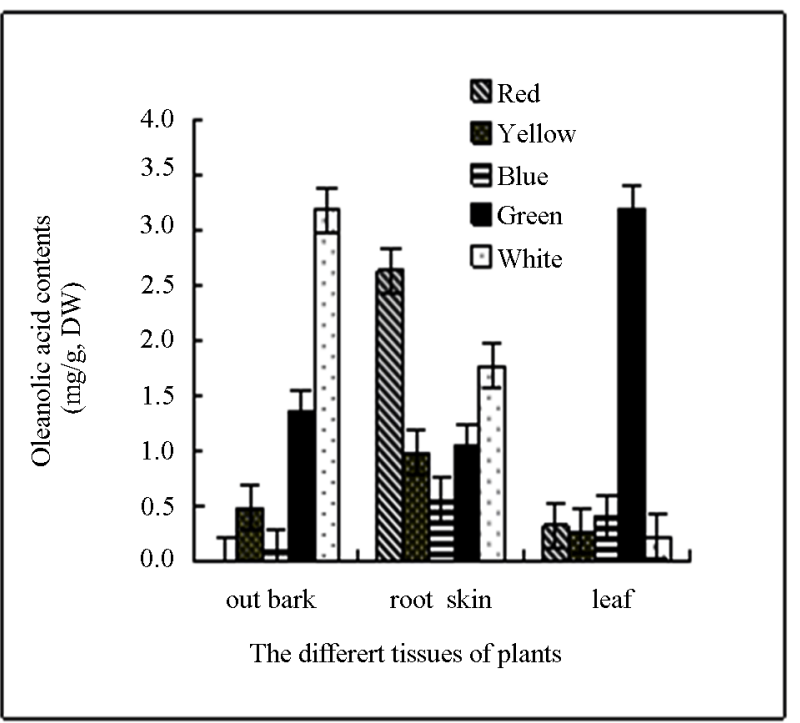

(a)

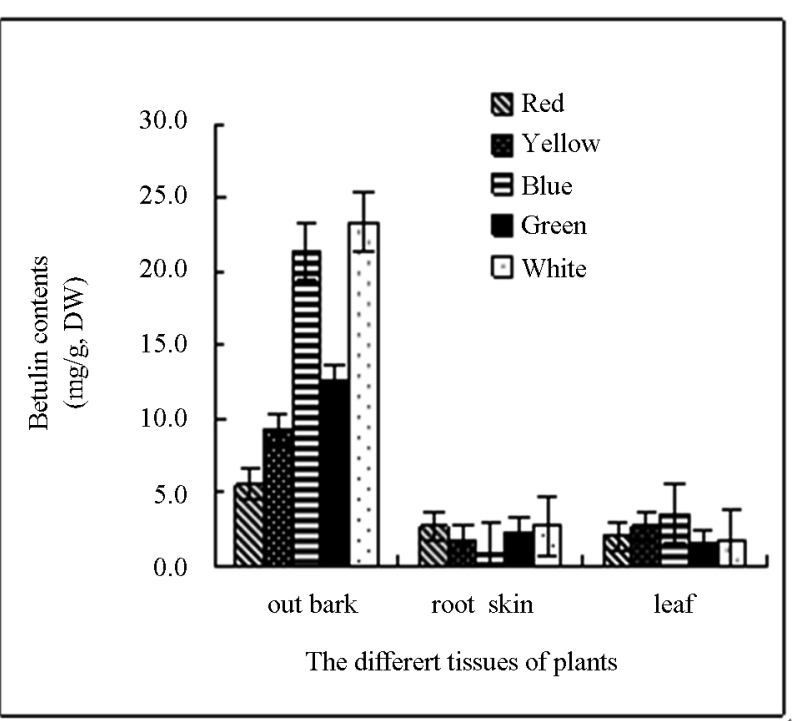

(b)

Figure 1. (a) and (b) The content and distributing of oleanolic acid and betulin in different tissues of the white birch plants treated with different lights. $\mathrm{Y}$-axis indicates the concentration of betulin. $\mathrm{X}$-axis indicates the different tissues of plants. 
mainly found in the cork layer of licorice root. Studies on the accumulation and distribution of oleanolic acid in birch have not been reported. In this study, we first investigated the accumulation of oleanolic acid in white birch saplings. We found that the highest contents of betulin and oleanolic acid were observed in the birch bark, followed by the root bark. The minimum content of betulin and oleanolic was observed in the leaf. The content of secondary metabolites significantly changed due to seasonal variation. The accumulation peak of oleanolic acid and betulin in stem bark and root bark of white birch appears in the August. The highest level of oleanolic acid and betulin in the stem bark of plants can reach approximately $8 \%$ and $3 \%$, respectively, of the plant dry weight. A small quantity of betulin and oleanolic acid can be detected in the leaves in August and September. The content of both substances was very low in other months or even cannot be detected. Changes of secondary metabolites in the growing season may be caused by the geographical location of Heilongjiang Province. Heilongjiang Province is located in northern China, where it is warm in the summer and cold in the winter. August is the hottest month of the year with the most abundant precipitation. Plant grows rapidly and the secondary meta- bolites are increased significantly in this month (Figure 2).

\subsection{The Effects of Light Intensity on the Accumulation Oleanolic Acid and Betulin Content in the out Bark of Birch}

Green plants create tissues through photosynthesis. The tissues produce a wide range of metabolites by transportation and transformation. Thus, light is necessary for the formation and accumulation of the active ingredients of plants. Lighting conditions include different types of light, different intensity of light and different illumination time. The role of light in the regulation of secondary metabolites is not consistent in different medicinal plants. Shohael et al. showed that optimal biomass accumulation of Eleutherococcus senticosus in somatic embryos occurred under the condition of fluorescent (Fl) and mixed blue plus far-red $(\mathrm{Bl}+\mathrm{Fr})$ irradiation. Red light resulted in the highest accumulation of eleutheroside E (51\%) and E1 (21\%), while fluorescent light produced the highest amount of total phenolic $(2.7 \%)$, total flavonoid (34\%) and chlorogenic acid (14\%) contents compared with the dark (control) grown mature embryos. $\mathrm{H}_{2} \mathrm{O}_{2}$, malondial-
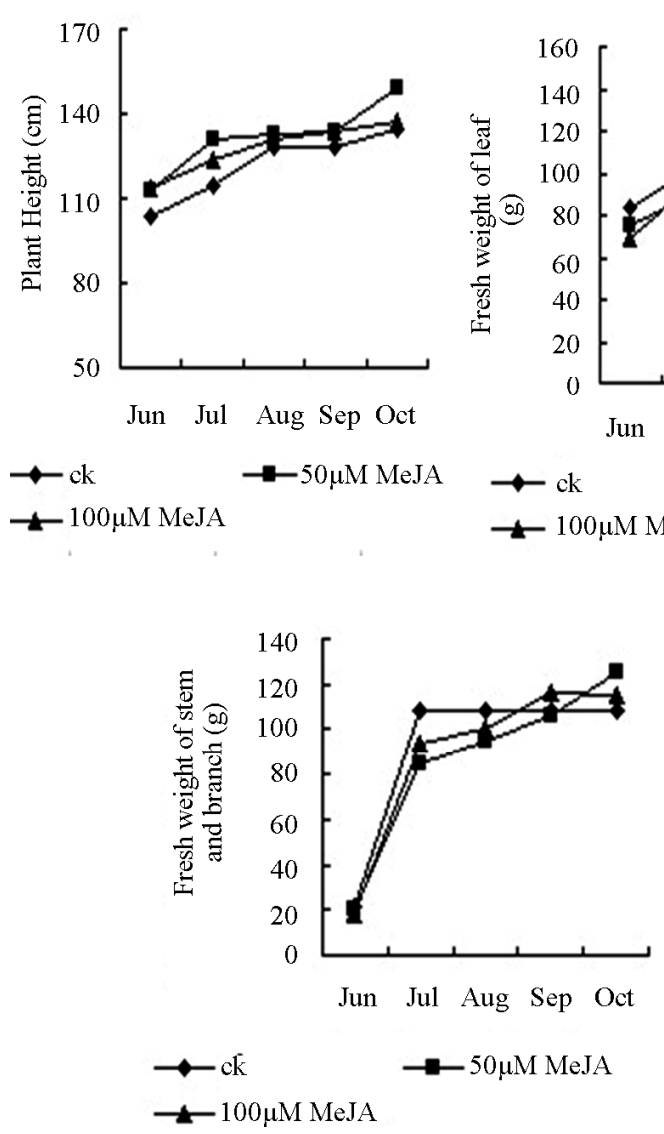
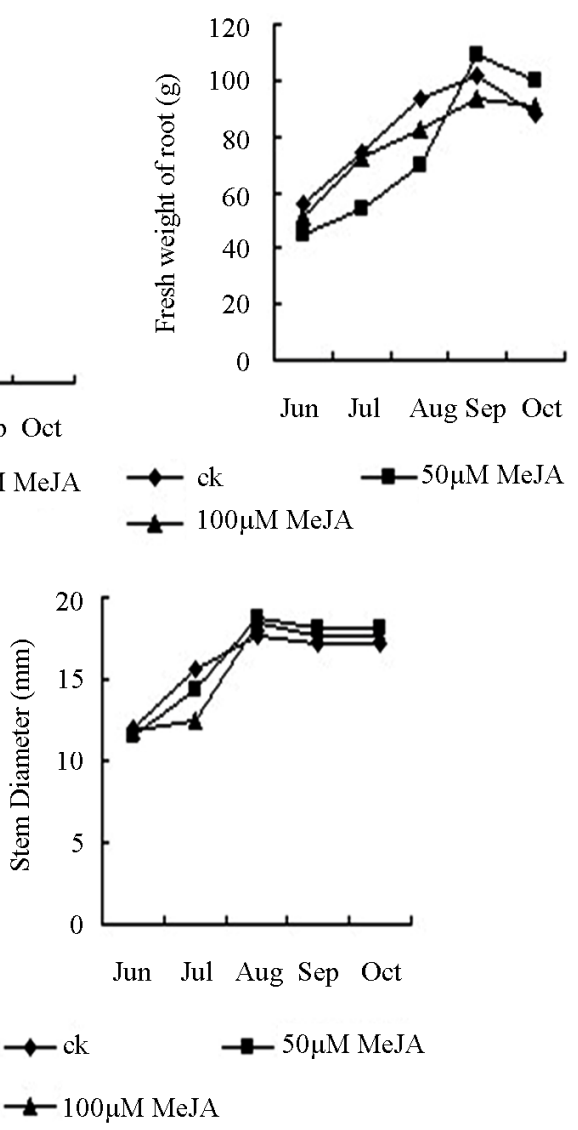

Figure 2. The growth change of different part of the birch plant under shade light and the whole light during growing season. 
dehyde (MDA) content and lipoxygenase (LOX) activties was increased in the red light-treated embryos compared with those in the dark (control) grown embryos [19]. Similar to the present studies, stimulatory effects of light on the formation of secondary metabolites have been reported in Perilla frutiescnens and Artimisia annua [29]. For some sun medicinal plants, increases in the light intensity can improve the content of metabolites. For instance, chlorogenic acid content in the honeysuckle grown in sunny condition was higher than that grown in the slope with less sunlight [30]. The atropine content in belladonnae cultivated in the open air accounted for $0.7 \%$ of those cultivated in overshadow conditions. High content of flavonoids was observed in pentaphyllum cultivated under light conditions. Shading can reduce the light intensity. Previous studies found that ginsenoside content accounted for $4.5 \%$ of the dry weight when arbores were treated with $20 \%$ light transmission [31]. Shading also plays a significant role in regulating guercetin content in the leaves of Ginkgo biloba saplings. Camptothecin content in leaves of Camptotheca seedling was elevated with increased degree of shading. However, serious shading ( $20 \%$ light intensity of the total light) treatment at the late stage (at day 75) decreased the content of camptothecin [32]. This is probably due to the short of normal products of photosynthesis leading to the disruption of basic physiological metabolism. More studies are needed to reveal the relationship between the optical quality and secondary metabolites, especially the relationship between UV-B and phenolic category, terpenoids and flavonoids. It is generally believed that enhanced UV radiation can induce plants to produce more phenolic substances. The highest content of cardiac glycosides in digitalis was achieved by blue light irradiation, while the content was very low under the yellow light, red light, green light and dark conditions. When the self-support-type cell lines of Catharanthus roseus was cultivated in the liquid medium, the ability of red light to in- crease the alkaloid synthesis was higher than that of blue light. Blue light has the strongest role in promoting flavonoid synthesis in callus of Saussurea medusa Maxim, followed by the far-infrared light and white light treatment. The red light has the lowest ability to promote flavonoid synthesis [33].

Our study result that accumulation of oleanolic acid and betulin in the stem bark in August was significantly higher than that in other months. In addition, the content of oleanolic acid and betulin in the birch with shading treatment was 26.419 and $81.023 \mathrm{mg} \cdot \mathrm{g}^{-1}$, respectively, which was improved by $45.09 \%$ and $30.50 \%$ compared with those in the birch with non-shading treatment (Figure 3). Secondary metabolites were accumulated slightly in leaves and root bark during the growing season. Oleanolic acid and betulin in leaves and betulin in root bark of birch with shading treatment were higher than that with non-shading treatment. The content of both betulin and oleanolic acid was very low in other months (October). Triterpenes cannot be detected in the leaves and thus, the data were not shown. From June to August, the plant grew gradually. The fresh weight of birch with shading light treatment was lower than the control birch. In September and October, the weather in Haerbin became cold and the leaves started falling. Therefore, the fresh weight of the plant was reduced. The reduction in the shading-treated plants was higher than the plants with the non-shading treatment (Figure 4).

Our study found that red, yellow, blue, green light treatment can promote the content of triterpenoid in the birch leaves, but they significantly reduced the accumulation of oleanolic acid and betulin in the stem skin. It is worth to note that green light can promote oleanolic acid in leaf, while blue light can promote the accumulation of betulin. In addition, our study found that leaf wilted after a variety of light treatment, which seriously inhibited plant and biomass growth within one month (data not shown). These results indicated that photosynthesis in
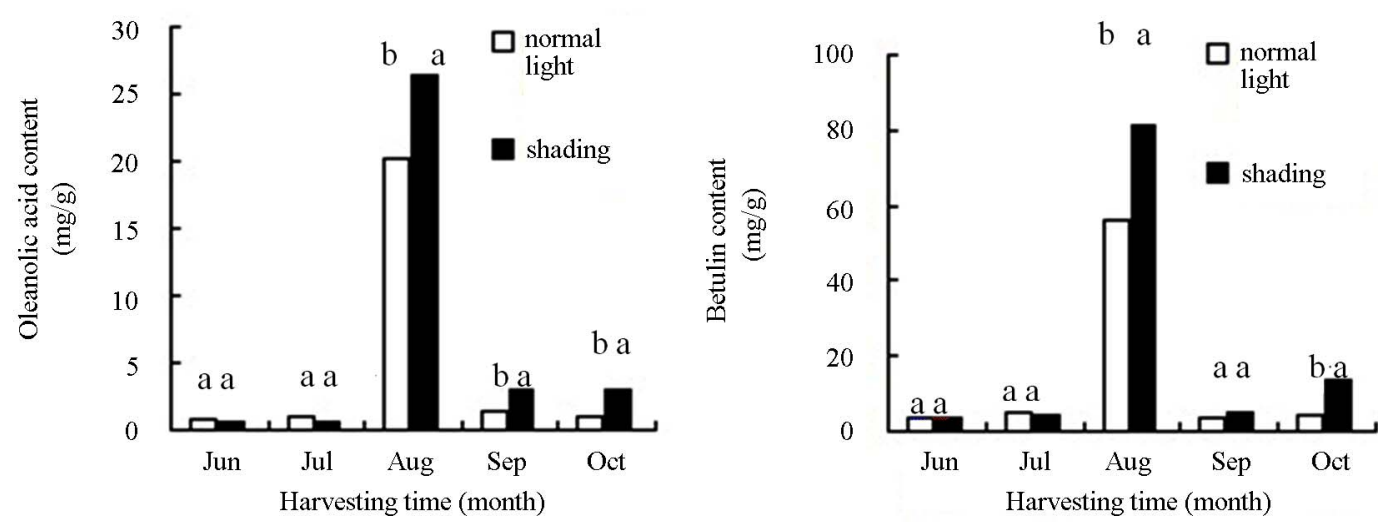

Figure 3. The effects of light intensity on the accumulation oleanolic acid and betulin content in out bark of birch plants. 

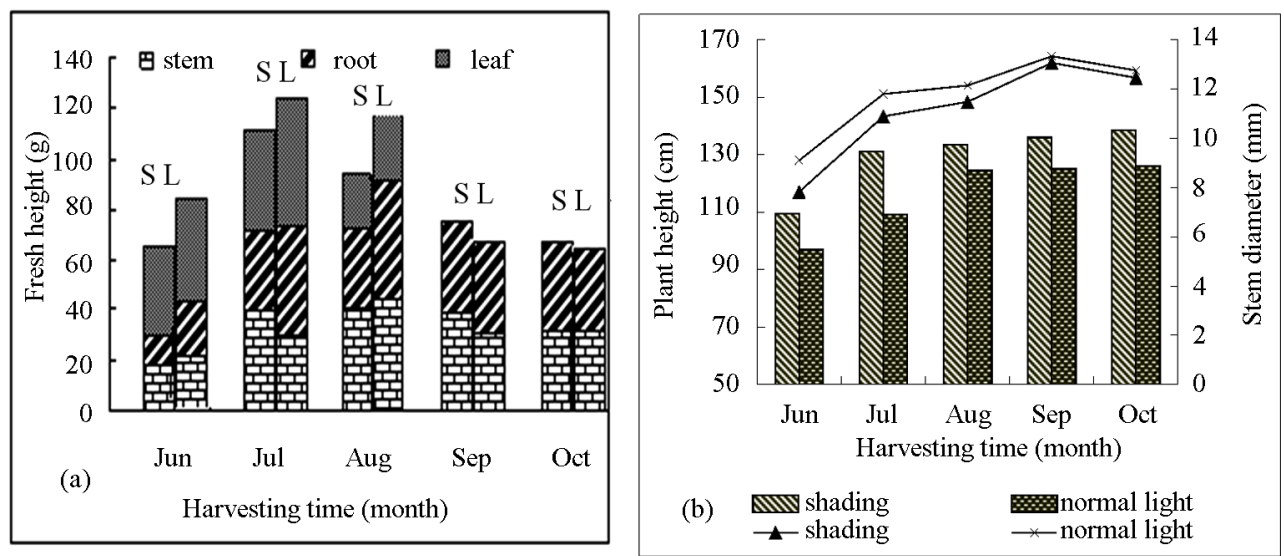

Figure 4. The growth change of different part of the birch under shading light and the whole light during growing season. (a) Includes stems, leaves, root fresh weight; (b) Includes plant height, stem diameter; S: shading (50\% of the normal light); L: normal light.

leaves was restricted by light treatment and dry matter accumulation was reduced, leading to a low level of total amount of secondary metabolites in plants. This is the reason why this experiment lasted only for one month after the light treatment. Compared with the full sunshine, appropriate shading treatment (light transmittance 50\%) inhibited plant growth (Figures 4 and 5), but significantly promoted the accumulation of secondary metabolites in stem bark, leaves and roots of the plant. These results indicated that accumulation of secondary metabolites product and plant growth require the most appropriate environmental conditions. This is also similar to previous results showing that coercion ecological environment is not conducive to primary metabolism, but can facilitate the accumulation of secondary metabolites of medicinal plants (sun). Therefore, we should select an appropriate cultivation condition to maintain the balance between plant growth and accumulation of secondary products in medicinal plants.

In Figures 3, small letters indicated that the significantly difference of different treatment in the 0.01 level.

\subsection{The Effect of Meja on the Accumulation of Oleanolic Acid and Betulin in White Birch Saplings}

Methyl jasmonate (MeJA) is an odoriferous compound in essential oils of the frangipani flower from jasmine (Jasminum). Volatile MeJA has been proven to be used for chemical communication between different species or same species of plants. MeJA can be spread in the air and induce the synthesis of chemical substances for plant defense system [34]. MeJA is a very effective elicitor inducing the synthesis of a variety of secondary metabolites $[35,36]$. Previous studies showed that that exogenous JA and/or MeJA can induce a set of defense genes and synthesis of defense compounds. The genes that can be induced by MeJA include those encoding plant defensins and thionins [37], phytoalexins [38], PR-proteins [39] and proteinase inhibitors [40]. A number of secondary metabolites were accumulated in cultured cells of various plant species upon treatment with JA. Studies also found that exogenous MeJA can induce the synthesis of anti-bacterial substances, allelochemicals terpenoid and diterpenes (which are important anti-cancer drugs) in rice leaves. MeJA induces the synthesis of triterpenoid precursor squalene (SE) in culture licorice (Glycyrrhiza glabra) and ginseng. MeJA can also promote the expression of $\beta-A S$ and ginsenoside synthase genes [24,41]. At the same time, MeJA promotes the synthesis of many monoterpene and terpenoid resin from spruce, pine and coconut to resist pathogen infection and insect bite $[26,42,43]$.

In our study, Oleanolic acid comes mainly from the skin of white birch stems. An inverted "V"-shaped dynamic curve of oleanolic acid accumulation in stem bark was observed in different months (Figure 6). The peak accumulation occurred in the early August. Treatment with MeJA can promote synthesis of oleanolic acid in the plant. The order of the MeJA effect on oleanolic acid content in the stem bark was $100>50 \mu \mathrm{M} \cdot \mathrm{L}^{-1}>\mathrm{ck}$ (control). The oleanolic acid content in plants treated with 100 or $50 \mu \mathrm{M} \cdot \mathrm{L}^{-1} \mathrm{MeJA}$ was 2.416 and 1.942 times, respectively, of the control plants (Figure 6).

Accumulation peak of oleanolic acid in leaves appeared at early September. The content of oleanolic acid in the leaves of birch treated with 100 or $50 \mu \mathrm{M} \cdot \mathrm{L}^{-1}$ MeJA was 6.3 and 4.23 times, respectively, of that in the control plants. Triterpenoid accumulation was not detected in the leaves in June, July and October. Oleanolic acid in the root bark was accumulated rapidly in July and August. In addition, the oleanolic content in the root bark 


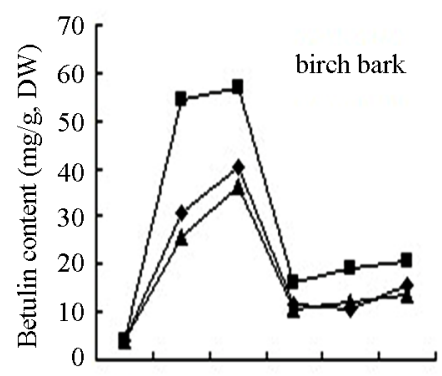

(a) May Jun Jul Aug Sep Oct

$\rightarrow 50 \mu \mathrm{M} \mathrm{MeJA} \rightarrow-100 \mu \mathrm{M}$ MeJA $\rightarrow \mathrm{ck}$

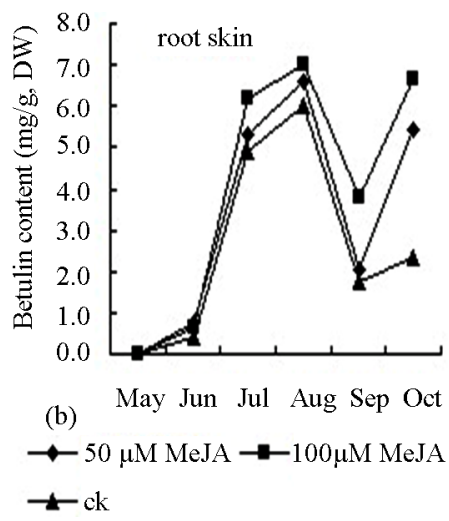

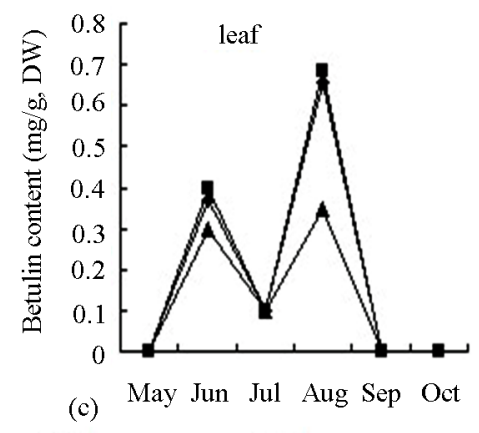

$\rightarrow-50 \mu \mathrm{M} \mathrm{MeJA} \rightarrow-100 \mu \mathrm{M} \mathrm{MeJA}$
$\rightarrow$ ck

Figure 5. Effect of MeJA on accumulation of betulin content in bark (a), root skin (b), and leaf (c) of birch saplings.
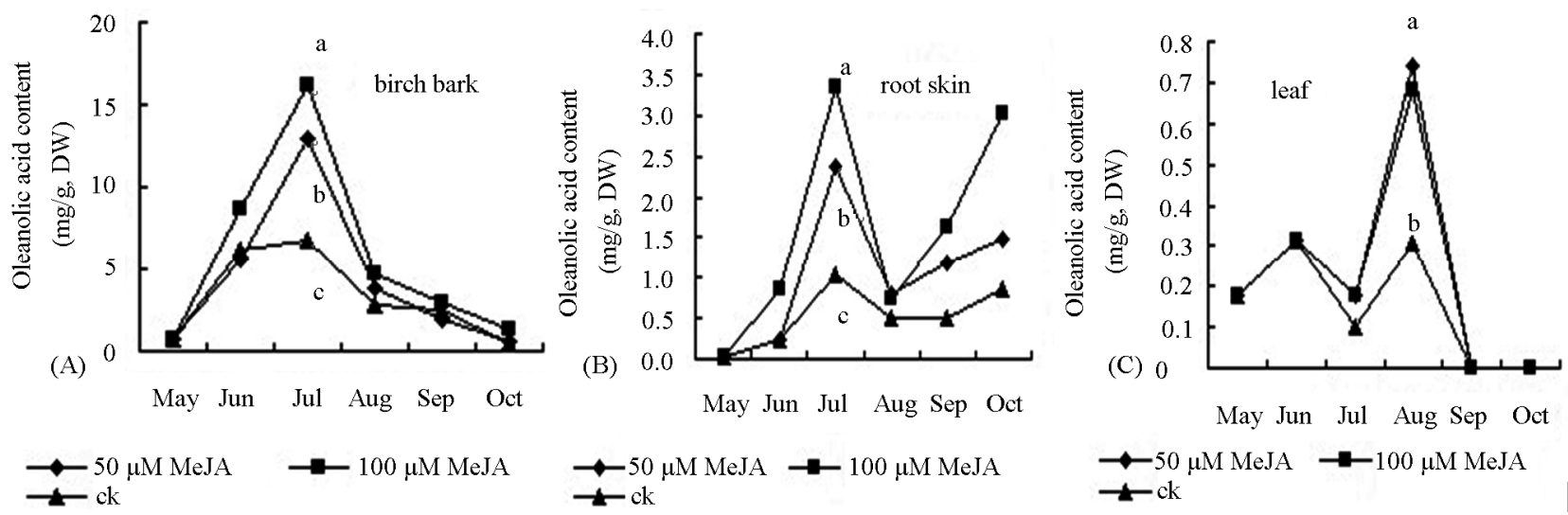

Figure 6. Effect of MeJA on accumulation of oleanolic acid content in bark (A), root skin (B) and leaf (C) of birch saplings.

of birch treated with 100 or $50 \mu \mathrm{M} \cdot \mathrm{L}^{-1} \mathrm{MeJA}$ was 3.33 and 2.21 times, respectively, of that in the control plants. Accumulation of oleanolic acid declined after August and then increased in the root in September. Throughout the growing season, the effect of $100 \mu \mathrm{M} \cdot \mathrm{L}^{-1} \mathrm{MeJA}$ was larger than that of $50 \mu \mathrm{M} \cdot \mathrm{L}^{-1} \mathrm{MeJA}$ on oleanolic acid accumulation in leaves and root bark. Betulin is mainly originated from the stem skin of white birch. Since early June, betulin began rapid accumulation. Treatment with $100 \mu \mathrm{M} \cdot \mathrm{L}^{-1}, 50 \mu \mathrm{M} \cdot \mathrm{L}^{-1}$ or ck MeJA resulted in the betulin accumulation rate of $0.887,0.607$ and 0.543 $\mathrm{mg} \cdot \mathrm{d}^{-1}$, respectively (Figuer 5). Accumulation peak of betulin was achieved in early August. The content of betulin in the birch treated with $100 \mu \mathrm{M} \cdot \mathrm{L}^{-1} \mathrm{MeJA}$ was significantly higher than that treated with $50 \mu \mathrm{M} \cdot \mathrm{L}^{-1}$ MeJA or ck. Betulin content started to decline in the stem bark. The accumulation peak of betulin in the leaves occurred in early September. With two concentrations of MeJA treatment, betulin content in the leaves was 2 times of the control plants. In other reproductive period, betulin accumulation in the leaf of was very low or cannot be detected. Rapid accumulation of betulin in the root bark started in early July. Betulin content in the birch with MeJA treatment was significantly higher than that in the control plants in August and September. Degradation of secondary metabolites started after September. In the growing season between June and September, the fresh leaf weight of birch treated with MeJA was decreased compared with the control plants. The majority of the leaves were detached from the plants in October. Growth of root and stem was inhibited from June to August. However, in September and October, the fresh weight of the roots and stems branches in birch treated with MeJA were higher than the control plants. The fresh weight of intact plant changed similarly in different seasons (Figure 2).

In this study, we found that triterpene in the leaf of MeJA-treated birch was significantly higher than that in the control plants in August and September. Triterpene content was very low or undetectable in other months. It is worth to note that at the growth season, for example, from June to September, leaf fresh weight of MeJAtreated plants was reduced compared with the control plants. At the same time, the root and branch growth was inhibited from June to August. However, from September to October, fresh weight of the roots and stems was higher in the MeJA-treated plants than that in the controls (Figure 2). These results indicated that that early 
MeJA treatment inhibited the growth of plants, but MeJA still induced the synthesis of defense substances (Phytoalexin) and accumulation of secondary metabolites in order to resist the gradual cooling of the air temperature and enhances the plant resistance. Therefore, the fresh weight of branch stem, root and whole plant in late stage of MeJA-treated plant were higher than those during the growing season.

\section{Experimental Section}

\subsection{Plant Material}

In 2007, birch seedlings were planted in the greenhouses of Maoershan test base of Northeast Forestry University (Haerbin, China). In May 2008, the white birch seedlings were transplanted to plastic pot with a diameter of $25 \mathrm{~cm}$, a high of $35 \mathrm{~cm}$ and a soil weight of $10 \mathrm{~kg}$. The nursery soil has a peat: sand ratio of 2:1:1 (V/V/V). NPK compound fertilizer $\left(0.5 \mathrm{~kg} / \mathrm{m}^{3}\right)$ was applied. Repeated mixing was performed to ensure a uniform soil in the pot after sieving. Conventional management with the same quantity of watering in each pot was applied.

\subsection{Light Treatment}

Light treatment using red, blue, green and yellow filter was conducted in July in the greenhouse. A transparent plastic film was used as a control treatment. Each treatment was conducted for 3 to 5 single plant. Plants were harvested after 1 month of treatment with different light. Sub-stem bark, leaves and root bark samples were collected and dried until HPLC analysis. In early June, the plants were covered with shade net (shading treatment) in order to achieve $50 \%$ of the normal intensity. Nonshading treatment was used as a control. Samples were collected once every 30 days of treatment.

\subsection{MeJA Treatment}

Seedlings with consistent growth were chosen for MeJA treatment. MeJA (Sigma; St. Louis) was added with 95\% ethanol to promote melting and diluted in distilled water containing approximately $0.5 \%$ ethanol. Two concentrations of MeJA (100 and $50 \mu \mathrm{M} \cdot \mathrm{L}^{-1}$ ) was used for treatment. $0.5 \%$ ethanol aqueous solution was used as a control under the same conditions. MeJA-treated samples were collected every month. Each treatment was repeated for 3 plants were detected for contents of oleanolic acid and betulin of the stem bark; root and leaf by HPLC analysis.

\subsection{High Performance Liquid Chromatography (HPLC) Detection}

The contents of oleanolic acid and betulin were measured according to Zhao et al. [28] with some modifications. Dry samples were ground into fine powders and extracted with $95 \%$ ethanol at a ratio of $10: 1(\mathrm{v} / \mathrm{w})$ at $25^{\circ} \mathrm{C}$ with gentle shaking for $24 \mathrm{~h}$, followed by $10 \mathrm{kHz}$ strength of ultrasound treatment for $40 \mathrm{~min}$. After centrifugation at $10,000 \times g$ for $10 \mathrm{~min}$, the supernatant was filtered through a $0.45 \mu \mathrm{m}$ filter. The amount of oleanolic acid and betulin in each extract was quantified using a high performance liquid chromatograph (HPLC) (Waters 600 controller auto injector) with a C18 Inertsil 5 ODS-2 column $(4.6 \mathrm{~mm} \times 250 \mathrm{~mm})$ and a mobile phase of $88 \%$ acetonitrile $/ 12 \% \mathrm{H}_{2} \mathrm{O}$. The flow rate was $1 \mathrm{ml} \cdot \mathrm{min}^{-1}$, the injection volume was $20 \mu \mathrm{l}$ and the eluent was monitored at $210 \mathrm{~nm}$ using a Waters 717 photodiode array detector.

\subsection{The Statistical Analysis}

The DPS7.50 software and Duncan's method for difference between treatment significant variance analysis were used.

\section{Conclusions}

These results showed that betulin and oleanolic were accumulated mainly in the stalk skin. The content of both substances in the stalk skin was significantly affected by seasons with a peak accumulation in August.

The content of oleanolic and betulin was significantly decreased in the stem skin treated with 4 types of light (red, yellow, blue and green) compared with the plant with normal illumination. In contrast, oleanolic acid in leaves was increased by 13.28 folds when the white birch was treated with green light. Betulin was increased by 1.959 folds in leaves of white birch treated with blue light. The highest content of betulin and oleanolic acid in various organs of birch with appropriate shading treatment (light transmittance: $50 \%$ ) was increased by $45.09 \%$ and $30.50 \%$, respectively, in comparison with those with non-shading treatment.

Content of oleanolic acid and betulin can be significantly improved in various parts of birch after treatment with different concentration of MeJA. The content of oleanolic acid in the leaves of birch treated with 100 or $50 \mu \mathrm{M} \cdot \mathrm{L}^{-1} \mathrm{MeJA}$ was 6.3 and 4.23 times, respectively, of that in the control plants. In addition, the oleanolic content in the root bark of birch treated with 100 or 50 $\mu \mathrm{M} \cdot \mathrm{L}^{-1} \mathrm{MeJA}$ was 3.33 and 2.21 times, respectively, of that in the control plants. The accumulation peak of betulin in the leaves occurred in early September. With two concentrations of MeJA treatment, betulin content in the leaves was twice of the control plants. In other reproductive period, betulin accumulation in the leaf was very low or cannot be detected. The study lays the foundation to understand the synthesis mechanism and meta- 
bolic regulation of oleanolic acid and betulin in white birch.

\section{Acknowledgements}

We thank Peng Hong-Mei and Teng Wen-Hua of Maoershan Experimental Forestry Center for greenhouse management of Northeast Forestry University, Harbin, China. This work was supported by Funds for Personnel Training of National Basic Science (J1210053), Natural Science Foundation of Heilongjiang Province (C201110), Natural Science Foundation of China (31200428), and the Fundamental Research Funds for the Central Universities (China, DL12CA04).

\section{REFERENCES}

[1] R. Mukherjee, V. Kumar and S. K. Srivastava, "Betulinic Acid Derivatives as Anticancer Agents: Structure Activity Relationship," Anti-Cancer Agents in Medicinal Chemistry, Vol. 6, No. 3, 2006, pp. 271-279. http://dx.doi.org/10.2174/187152006776930846

[2] K. Yoshiki, S. Michiko and Y. Kimihisa, "Triterpenoids from the Floral Spikes of Betula platyphylla var. Japonica and Their Reversing Activity against Multidrug-Resistant Cancer Cells," Journal of Natural Products, Vol. 70, No. 4, 2007, pp.623-627. http://dx.doi.org/10.1021/np060631s

[3] E. H. Pisha, I. L. Chai and T. E. Chagwedera, "Discovery of Betulinic Acid as a Selective Inhibitor of Human Melanoma That Functions by Induction of Apoptosis," Nature Medicine, Vol. 1, No. 10, 1995, pp. 1046-1051. http://dx.doi.org/10.1038/nm1095-1046

[4] B. B. Saxena, L. Zhu and M. Hao, "Boc-Lysinate-Betulonic Acid: A Potent, Anti-Prostate Cancer Agent," $\mathrm{Me}$ dicinal Chemistry, Vol. 14, 2006, pp. 6249-6258.

[5] M. C. Aguirre, C. Delporte and N. Backhouse, "Topical Anti-Inflammatory Activity of 2 Alpha-Hydroxy Pentacyclic Triterpene Acids from the Leaves of Ugni molinae," Medicinal Chemistry, Vol. 14, No. 16, 2006, pp. 5673-5677. http://dx.doi.org/10.1016/j.bmc.2006.04.021

[6] T. Fujioka, Y. Kashiwada and R. E. Kilkuskie, "AntiAIDS Agents.11.Betulinic Acid and Platanic Acid as Anti-HIV Principles from Syzigium claviflorum, and the Anti-HIV Activity of Structurally Related Triterpenoids," Journal of Natural Products (Lloydia), Vol. 57, No. 2, 1994, pp. 243-247. http://dx.doi.org/10.1021/np50104a008

[7] L. Huang, K. H. Lee and C. H. Chen, "Synthesis and Anti-HIV Activity of Bi-Functional BA Derivatives," Bioorganic \& Medicinal Chemistry, Vol. 14, No. 7, 2006, pp. 2279-2289.

http://dx.doi.org/10.1016/j.bmc.2005.11.016

[8] N. G. Kyoko, Y. Koji and T. Masahiko, "Cancer Preventive Agents 9 Betulinic Acid Derivatives as Potent Cancer Chemopreventive Agents," Bioorganic \& Medicinal Chemistry Letters, Vol. 19, No. 13, 2009, pp. 3378-3381. http://dx.doi.org/10.1016/i.bmcl.2009.05.050
[9] A. Falamas, S. Cinta and C. A. Pinzaru, "Betulin and Its Natural Resource as Potential Anticancer Drug Candidate Seen by FT-Raman and FT-IR Spectroscopy," Journal of Raman Spectroscopy, Vol. 42, No. 1, 2011, pp. 97-107. http://dx.doi.org/10.1002/jrs.2658

[10] T. Tsuruga, Y. T. Chun and Y. Ebizuka, "Biologically Active Constituents of Melaleuca leucadendron: Inhibitors of Induced Histamine Release from Rat Mast Cells," Chemical \& Pharmaceutical Bulletin, Vol. 39, No. 12, 1991, pp. 3276-3278. http://dx.doi.org/10.1248/cpb.39.3276

[11] X. H. Ma, Y. C. Zhao and L. Yin, "Studies on the Effect of Oleanolic Acid on Experimental Liver Injury," Acta Pharmacologica Sinica, Vol. 17, No. 2, 1982, pp. 93-97.

[12] J. Liu, "Pharmacology of Oleanolic Acid and Ursolic Acid," Journal of Ethnopharmacology, Vol. 49, No. 2, 1995, pp. 57-68. http://dx.doi.org/10.1016/0378-8741(95)90032-2

[13] S. Balanehru and B. Nagarajan, "Protective Effect of Oleanolic Acid and Ursolic Acid against Lipid Peroxidation," Biochemistry International, Vol. 24, No. 5, 1991, pp. 981-990.

[14] S. Shibata, "Chemistry and Cancer Preventing Activities of Ginseng Saponins and Some Related Triterpenoid Compounds," Journal of Korean Medical Science, Vol. 16, 2001, pp. 28-37.

[15] F. Krewzaler and K. Hahlbrock, "Flavonoid Glycosides from Illuminated Cell Suspension Cultures of Petroselinum hortense," Phytochemistry, Vol. 12, No. 5, 1976, pp. 11491152. http://dx.doi.org/10.1016/0031-9422(73)85031-9

[16] J. J. Zhong, T. Seki and S. Kinoshita, "Effect of Light Irradiation on Anthocyanin Production by Suspended Culture of Perilla frutescens," Biotechnology and Bioengineering, Vol. 38, No. 6, 1991, pp. 653-658. http://dx.doi.org/10.1002/bit.260380610

[17] A. M. Shohael, M. B. Ali and Y. E. Hahn, "Effect of Light on Oxidative Stress, Secondary Metabolites and Induction of Antioxidant Enzymes in Eleutherococcus Senticosus Somatic Embryos in Bioreactor," Process Biochemistry, Vol. 41, No. 5, 2006, pp. 1179-1185. http://dx.doi.org/10.1016/j.procbio.2005.12.015

[18] J. J. Cheong and Y. D. Choi, "Methyl Jasmonate as a Vital Substance in Plants," Trends in Genetics, Vol. 19, No. 7, 2003, pp. 409-413. http://dx.doi.org/10.1016/S0168-9525(03)00138-0

[19] G. Arimura, R. Ozawa and T. Shimoda, "Herbivory-Induced Volatiles Elicit Defence Genes in Lima Bean Leaves," Nature, Vol. 406, 2000, pp. 512-515. http://dx.doi.org/10.1038/35020072

[20] H. Hiroaki, H. Pengyu and T. Satoko, "Differential Expression of Three Oxidosqualene Cyclase mRNAs in Glycyrrhiza glabra," Biological \& Pharmaceutical Bulletin, Vol. 27, No. 7, 2004, pp. 1086-1092. http://dx.doi.org/10.1248/bpb.27.1086

[21] B. Arturo, L. J. Espina and R. T. Isidro, "Effect of Methyl Jasmonate on Properties of Intact Tomatofruit Monitored with Destructive and Nondestructive Tests," Journal of Food Engineering, Vol. 80, No. 4, 2007, pp. 1086-1095. 
http://dx.doi.org/10.1016/j.jfoodeng.2006.09.001

[22] K. M. Nkembo, J. B. Lee and T. Hayashi, "Selective Enhancement of Scopadulcic Acid B Production in the Cultured Tissues of Scoparia dulcis by Methyl Jasmonate," Chemical \& Pharmaceutical Bulletin, Vol. 53, No. 1, 2005, pp. 780-782. http://dx.doi.org/10.1248/cpb.53.780

[23] Y. Y. Wang and X. H. Shang, "Effects of Field Light Intensity and Quality on Biomass and Salidroside Content in Roots of Rhodiola sachalinensis," Acta Ecologica Sinica, Vol. 24, No. 4, 2004, pp. 674-679.

[24] M. Diane, T. Dorothea and G. Jonathan, "Methyl Jasmonate Induces Traumatic Resin Ducts, Terpenoid Resin Biosynthesis, and Terpenoid Accumulation in Developing Xylem of Norway Spruce Stems," Plant Physiology, Vol. 129, No. 3, 2002, pp. 1003-1018. http://dx.doi.org/10.1104/pp.011001

[25] K. Yoshiki, S. Michiko and I. Yasumasa, "3-O-Glutaryldihydrobetulin and Related Monoacyl Derivatives as Potent Anti-HIV Agents," Bioorganic \& Medicinal Chemistry Letters, Vol. 14, No. 23, 2004, pp. 5851-5853. http://dx.doi.org/10.1016/j.bmcl.2004.09.033

[26] G. L. Zhao, W. D. Yan and D. Cao, "Simultaneous Determination of Betulin and Betulinic Acid in White Birch Bark Using RP-HPLC," Journal of Pharmaceutical and Biomedical Analysis, Vol. 43, No. 3, 2007, pp. 959-962. http://dx.doi.org/10.1016/j.jpba.2006.09.026

[27] H. Hiroaki, H. Pengyu and I. Kenichiro, "Cloning and Characterization of a cDNA Encoding b-Amyrin Synthase Involved in Glycyrrhizin and Soyasaponin Biosyntheses in Licorice," Biological \& Pharmaceutical Bulletin, Vol. 24, No. 8, 2001, pp. 912-916. http://dx.doi.org/10.1248/bpb.24.912

[28] C. Z. Liu, C. Guo and Y. C. Wang, "Effect of Light Irradiation on Hairy Root Growth and Artemisinin Biosynthesis of Artemisia Annual," Process Biochemistry, Vol. 38, No. 4, 2002, pp. 581-585. http://dx.doi.org/10.1016/S0032-9592(02)00165-6

[29] Q. Li, Q. Ren and Y. L. Zhang, "Effects of Ecological Environment, Collecting Stages and Storage Time on Chlorogenic Acid Content of Lonicera ferdinandii Franch," China Journal of Chinese Materia Medica, Vol. 18, 1994, pp. 594-595.

[30] S. P. Serbin, D. N. Dillaway and E. L. Kruger, "Leaf Optical Properties Reflect Variation in Photosynthetic Metabolism and Its Sensitivity to Temperature," Journal of Experimental Botany, Vol. 63, No. 1, 2012, pp. 489502. http://dx.doi.org/10.1093/jxb/err294

[31] Z. A. Zhang, K. Z. Xu and Y. Y. Ren, "Effect of Light intensity on Content of Soluble Sugar, Starch and Ginseng Saponin in Ginseng Plant," Journal of Jilin Agricultural University, Vol. 16, No. 3, 1994, pp. 15-17.

[32] Y. Wang, S. J. Dai and X. F. Yan, "Effects of Light Intensity on Secondary Metabolite Camptothecin Production in Leaves of Camptotheca acuminata Seedlings,"
Acta Ecologica Sinica, Vol. 24, No. 6, 2004, pp. 1118 1122.

[33] Y. T. Zhou, "Studies on the Effect of Different Light Intensity on the Photosynthesis and Secondary Metabolit of Catharanthus Roseus," Master's Thesis, Southwest University, Chongqing, 2008.

[34] G. Shen, Y. Z. Pang and W. S. Wu, "Molecular Cloning, Characterization and Expression of a Novel JasmonateDependent Defensin Gene from Ginkgo biloba," Journal of Plant Physiology, Vol. 162, No. 10, 2005, pp. 11601168. http://dx.doi.org/10.1016/j.jplph.2005.01.019

[35] P. H. Bart, J. Thommaa and K. Eggermonta, "Disease Development of Several Fungi on Arabidopsis Can Be Reduced by Treatment with Methyl Jasmonate," Plant Physiology and Biochemistry, Vol. 38, No. 5, 2000, pp. 421-427. http://dx.doi.org/10.1016/S0981-9428(00)00756-7

[36] S. Kondo, S. Setha and D. R. Rudell, "Aroma Volatile Biosynthesis in Apples Affected by 1-MCP and Methyl Jasmonate," Mattheis Postharvest Biology and Technology, Vol. 36, No. 1, 2005, pp. 61-68. http://dx.doi.org/10.1016/j.postharvbio.2004.11.005

[37] F. R. Terras, K. Eggermont and V. Kovaleve, "Small Cysteine-Rich Antifungal Proteins from Radish: Their Role in Host Defense," Plant Cell, Vol. 7, No. 5, 1995, pp. 573-588.

[38] L. I. Il'inskaya, E. V. Goenburg and G. I. Chalenko, "Involvement of Jasmonic Acid in the Induction of Potato Resistance to Phytophthora Infection," Russian Journal of Plant Physiology, Vol. 43, No. 5, 1996, pp. 622-628.

[39] Y. Xu, L. D. Chang and D. Liu, "Plant Defence Genes Are Synergistically Induced by Ethylene and Methyl Jasmonate," Plant Cell, Vol. 6, No. 8, 1994, pp. 10771085.

[40] R. A. Creelman and J. E. Mullet, "Biosynthesis and Action of Jasmonates in Plants," Plant Molecular Biology, Vol. 48, No. 1, 1997, pp. 355-381.

[41] H. Hiroaki, H. Pengyu and T. Satoko, "Differential Expression of Three Oxidosqualene Cyclase mRNAs in Glycyrrhiza glabra," Biological \& Pharmaceutical Bulletin, Vol. 27, No. 7, 2004, pp. 1086-1092. http://dx.doi.org/10.1248/bpb.27.1086

[42] M. A. Naoumkina, L. V. Modolo and D. V. Huhman, "Genomic and Coexpression Analyses Predict Multiple Genes Involved in Triterpene Saponin Biosynthesis in Medicago truncatula," The Plant Cell, Vol. 22, No. 3, 2010, pp. 850-866.

http://dx.doi.org/10.1105/tpc.109.073270

[43] H. Zhang, M. Shibuya and S. Yokota, "Oxidosqualene Cyclases from Cell Suspension Cultures of Betula platyphylla var. Japonica: Molecular Evolution of Oxidosqualene Cyclases in Higher Plants," Biological \& Pharmaceutical Bulletin, Vol. 26, No. 5, 2003, pp. 642-650. http://dx.doi.org/10.1248/bpb.26.642 